\title{
Effect of Respiratory Exercises on the Prevalence of Atelectasis in Patients Undergoing Coronary Artery Bypass Surgery
}

\author{
Khodayar Oshvandi ${ }^{1}$, Atefeh Bostanbakhsh², Mohsen Salavati ${ }^{3 *}$, Mohammadhossein Bakhshaii ${ }^{4}$, \\ Abas Moghimbeighi' ${ }^{5}$ Zahra Maghsoudi6
}

1. Maternal and Child Care Research Center, Faculty of Nursing \& Midwifery, Hamadan University of Medical Sciences, Hamadan, Iran

2. Master of Nursing, Student Research Committee, School of Nursing and Midwifery, Hama-dan University of Medical Sciences, Hamadan, Iran

3. Instructor, School of Nursing and Midwifery, Hamadan University of Medical Sciences, Hamadan, Iran

4. Associate Professor, Department of Anesthesiology, School of Medicine, Hamadan University of Medical Sciences, Hamadan, Iran

5. Professor, Department of Biostatistics, School of Health, Hamadan University of Medical Sciences, Hamadan, Iran

6. PhD Student in Nursing, Student Research Committee, Hamadan University of Medical Sciences, Hamadan, Iran

\begin{tabular}{|c|c|}
\hline Article Info & ABSTRACT \\
\hline $\begin{array}{l}\text { Received: 2019/05/20; } \\
\text { Accepted: 2019/07/14; } \\
\text { Published Online: 2019/03/27 }\end{array}$ & $\begin{array}{l}\text { Introduction: Patients with coronary artery bypass (CAB) surgery are at risk of } \\
\text { atelectasis. Use of respiratory exercises as an effective approach has had controversy } \\
\text { finding in } \mathrm{CAB} \text { patients. This study aimed to determine the effect of breathing } \\
\text { exercises on the prevention of atelectasis in patients undergoing coronary artery } \\
\text { bypass surgery. }\end{array}$ \\
\hline
\end{tabular}

10.30699/sjhnmf.27.6.432

Methods: In this randomized clinical trial, 80 patients with CAB surgery who referred to Ekbatan Hospital, Hamadan, Iran from July 2013 to May 2015 were randomly divided into two groups. In experimental group $(n=40)$, respiratory exercises (30 deep respiratory per hour for 3 days postoperatively) were performed

Original Article by patients, after full education and extubation along with routine care. In control group $(n=40)$, patients only received routine care. Chest $x$ ray was used to diagnose atelectasis. Data analysis was done by SPSS 17.

Use your device to scan and read the article online

Results: The two groups did not differ significantly in terms of personal characteristics such as age, sex, education, bmi and etc. $(P<0 / 05)$. The result of the Mann-Whitney test in order to compare the two groups of test and control for the occurrence of atelectasis, respiration and respiratory type showed a significant difference between the two groups at all hours after surgery $(P<0 / 001)$. These findings confirmed the effectiveness of respiratory exercises.

Conclusion: This study showed that breathing exercises can reduce the incidence of atelectasis. Therefore, applying this method as a routine care is recommended in patients with $\mathrm{CAB}$ surgery.

Keywords: Breathing exercises, Coronary artery bypass surgery, Atelectasis

Corresponding Information: Mohsen Salavati, School of Nursing and Midwifery, Hamadan University of Medical Sciences,

Hamadan, Iran. Email: salavati@umsha.ac.ir

Copyright $\odot$ ) 2020, This is an original open-access article distributed under the terms of the Creative Commons Attribution-noncommercial 4.0 International License which permits copy and redistribution of the material just in noncommercial usages with proper citation.

How to Cite This Article:

Oshvandi K, Bostanbakhsh A, Salavati M, Bakhsai M, Moghimbeighi A, Maghsoudi Z. Effect of Respiratory Exercises on the Prevalence of Atelectasis in Patients Undergoing Coronary Artery Bypass Surgery. Avicenna J Nurs Midwifery care. 2019; 27 (6) :432-440 


\title{
بررسى تأثير تمرينات تنفسى بر بروز آتلكتازى در بيماران كانديد جراحى باىيس عروق كرونر \\ خدايار عشوندى'، عاطفه بوستان بخش؟، محسن صلواتى "، محمدحسين بخشايى؟،

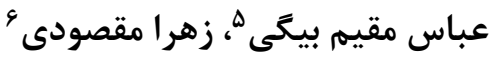

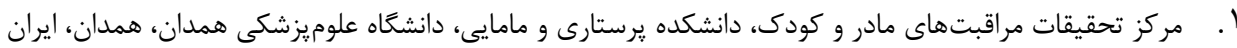

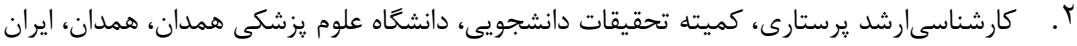

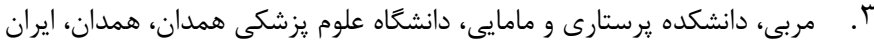

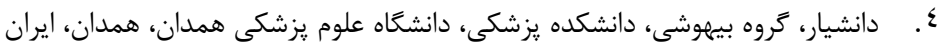

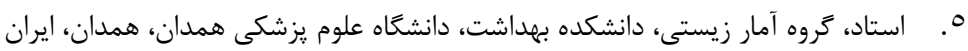

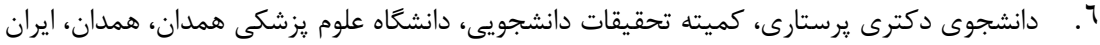

\begin{tabular}{|c|c|}
\hline קكيده & اطلاعات مقاله \\
\hline 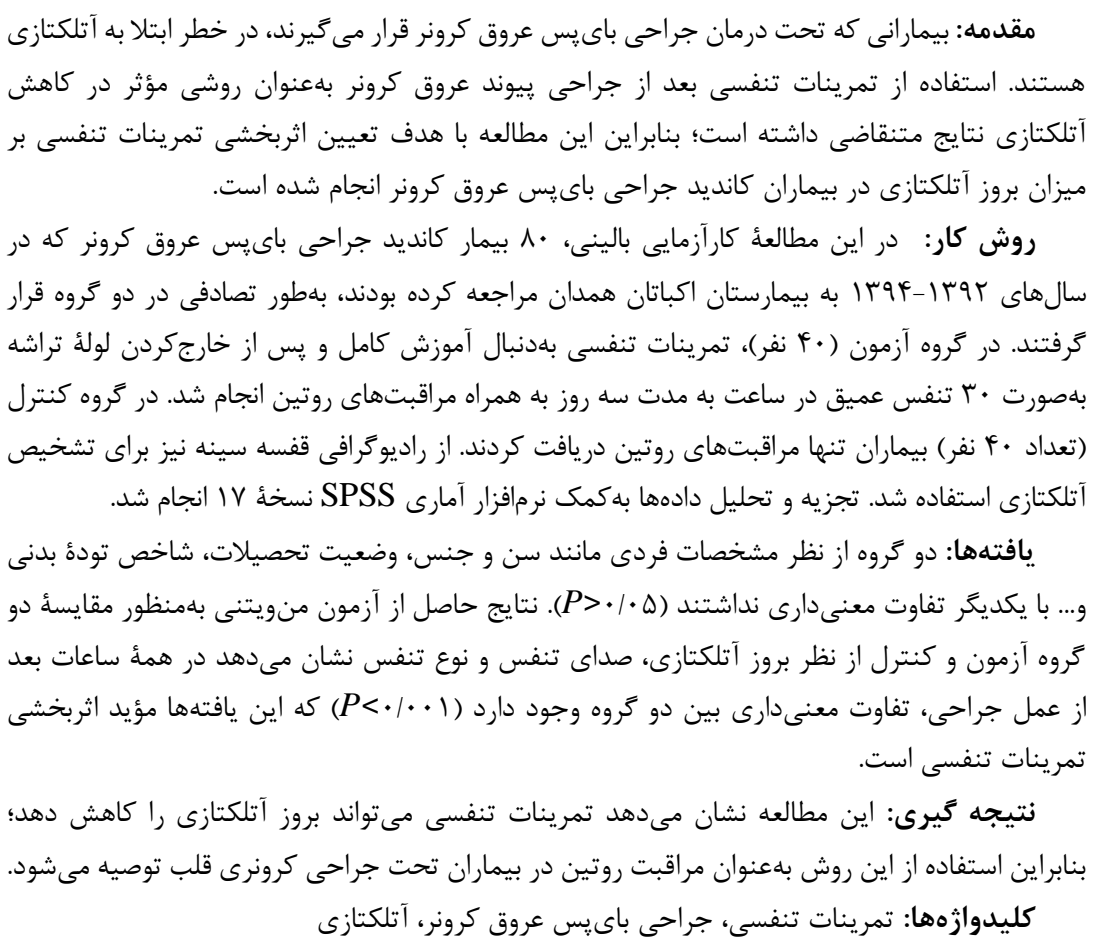 & 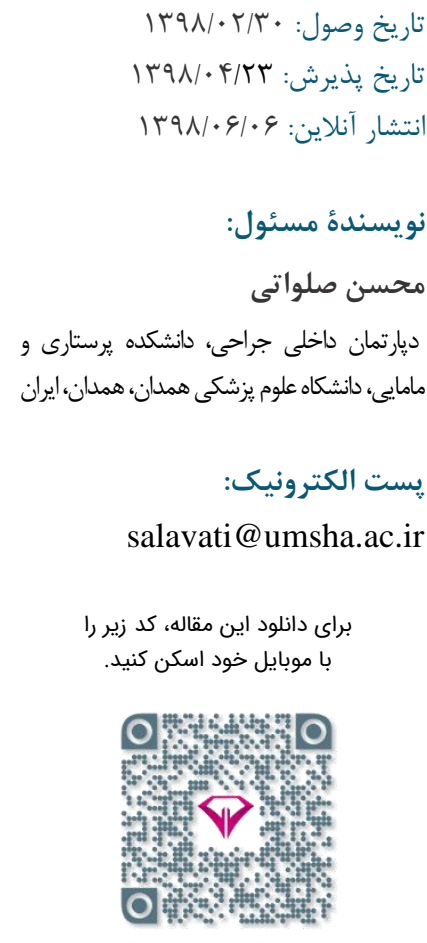 \\
\hline
\end{tabular}

جراحى باىيس عروق كرونر است [9، V]. آتلكتازى عامل

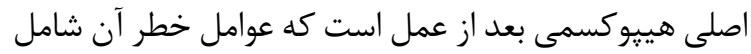

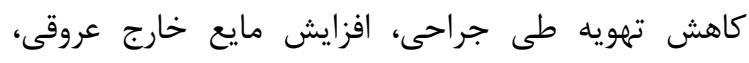

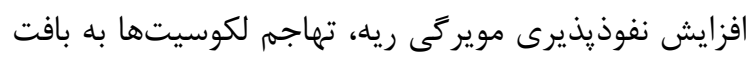

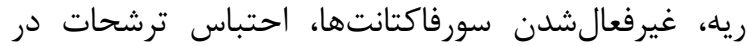
قاعدة ريه، كاهش ظرفيت باقى مانده عملى ريه و تغيير شكل قفسه سينه و ديافر اكم بهدليل استفاده از داروهاى شل كنندة عضلانى، برش استخوان جناغ و باىيس قلبى-ريوى است

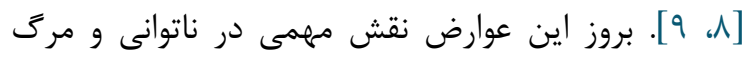

جراحى باىيس عروق كرونر بهعنوان درمان انتخابى براى بسيارى از بيماران دجار بيمارى عروق كرونر مطرح

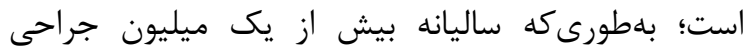
باى يس عروق كرونر در سراسر دنيا انجام مىشود [1، ب].

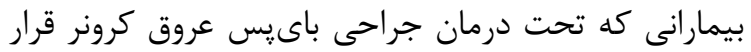

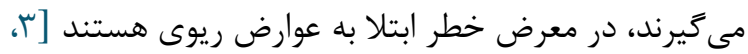

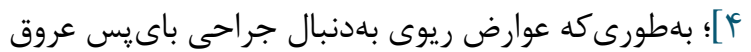

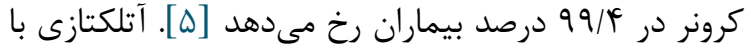

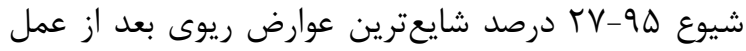


و كتبى از آنها، با يرتاب سكه بهصورت تصادفى در دو كروه

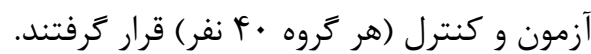

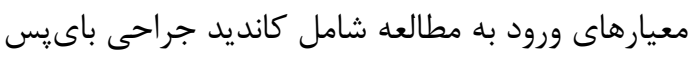

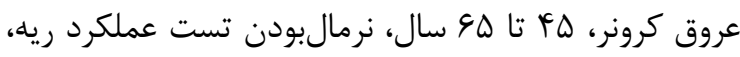
نبود بيمارى هاى حاد و مزمن ريوى و نرمال بودن عكس قفسأ

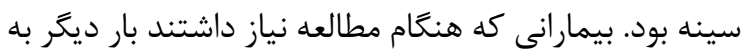

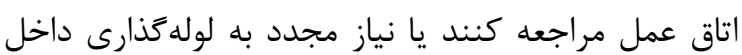

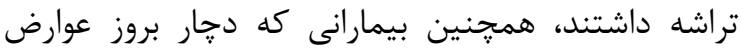
ناخواسته بعد از عمل (ينوموتوراكس، آمبولى ريه، آدم ريه،

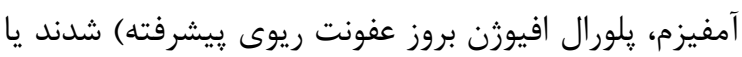

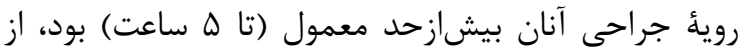
مطالعه خارج شدند. يزوهشكر يس از دريافت كد اخلاق به شماره شناسه

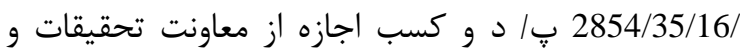
فناورى بهمنظور انجام يزوهش، به بيمارستان اكباتان مراجعه

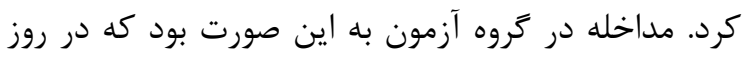

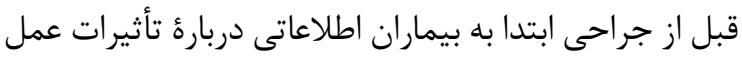
جراحى باىيس عروق كرونر بر عملكرد ريوى داده شد. سيس دستورالعمل انجام تمرينات تنفسى شامل انجام تنفس برى

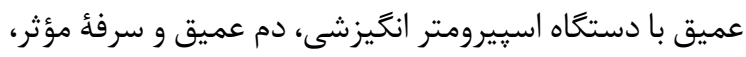
به آنها به آموزش داده شد. اين تمرينات يك ساعت يس إن از

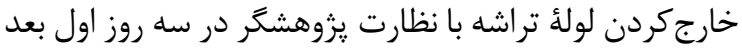

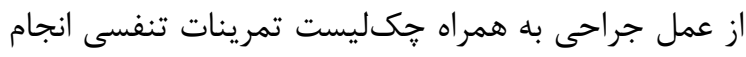

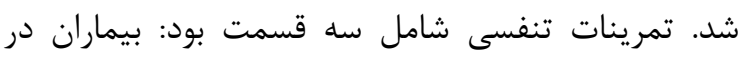

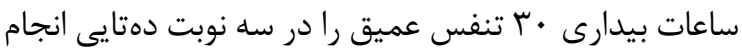

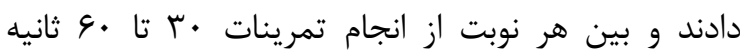

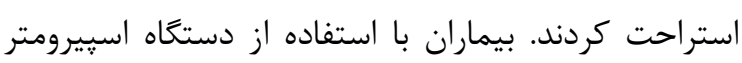
انخَيزشى، • ا تنفس عميق در هر ساعت از بيدارى انجام دادند. بيماران در وضعيت نشسته يس از انجام اندام دم عميق،

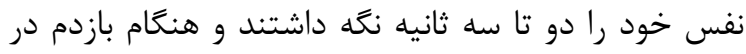

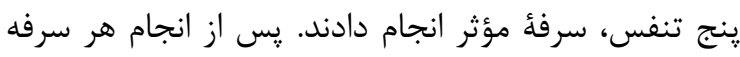

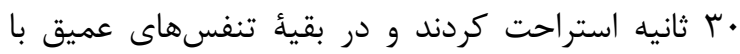

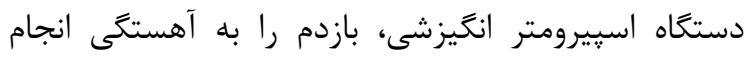

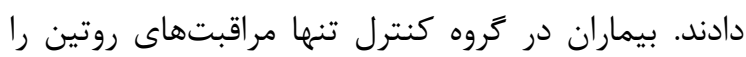

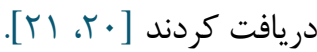
دادهها با استفاده از فرمهاى مشخصات دموَّر افيك و فرم

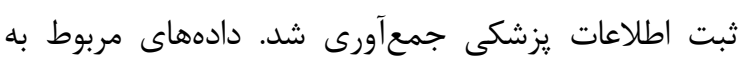

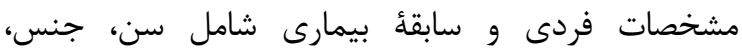

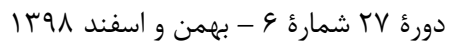

بيماران دارد و با افزايش اقامت در بيمارستان منجر به صرف

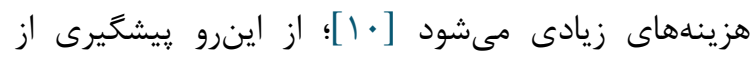

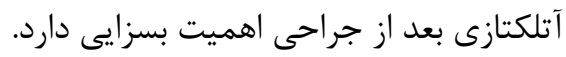

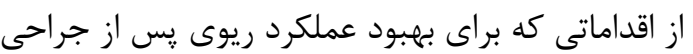
باى يس عروق كرونرى انجام مىشود، مى توان به فيزيوترايى

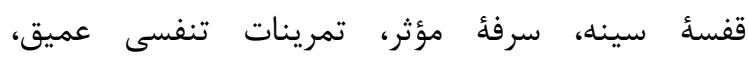
اسييرومترى انكَيزشى، فشار مثبت متناوب يا مداوم در زمان

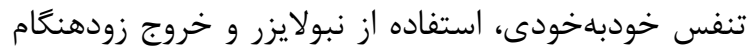

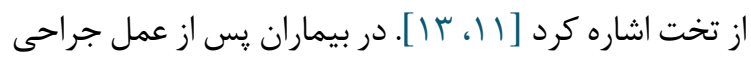

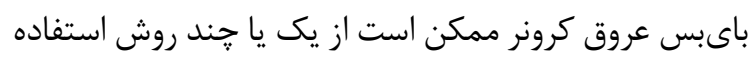

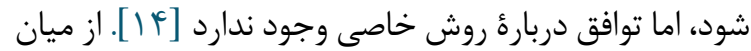
اين روشها، تمرينات تنفسى عميق، سرفئ مؤثر و استفاده از

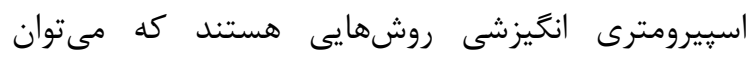
بلراحتى، بدون عارضه و با هزينهُ كم به بيماران كانديد

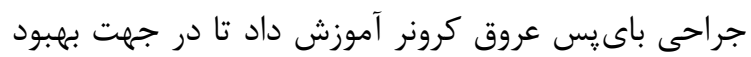

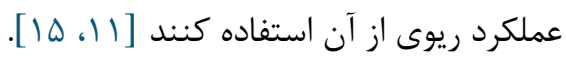
تحقيقات زيادى دربارة اثربخشى تمر ينات تنفسى انجان انجام شده است، اما نتايج آنها تفاوتهاى فراوانى با يكديكر دارند؛ بلهورى كه حتى مىتوان اين نتايج را در دو سر يكى طيف إنفائ

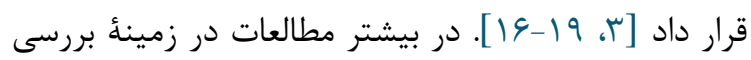
اثر تمرينات تنفسى، اين تمرينات با كيفيت و كميت متفاوت

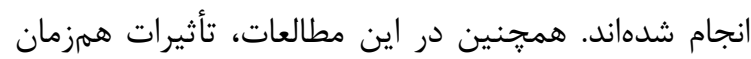

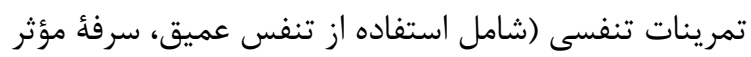

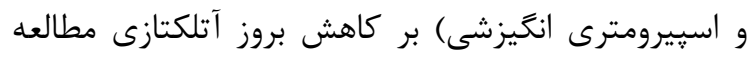

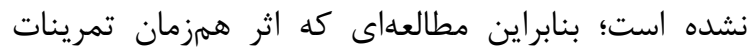

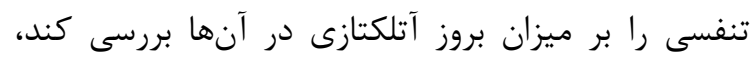

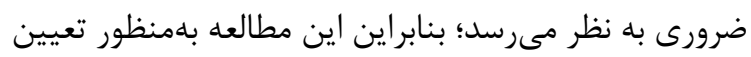

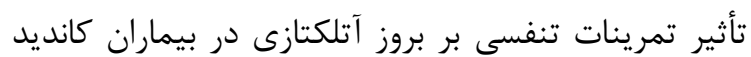
جراحى باىيس عروق كرونرى انجام شد.

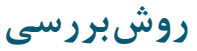

مطالعُ حاضر كارآزمايى بالينى همراه با گروه كنترل،

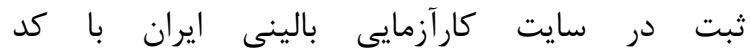
IRCT201112188451N1 بيماران كانديد جراحى باىيس عروق كرونر بسترى در بيمارستان اكباتان همدان، تنها مركز جراحى قلب شئ شهر

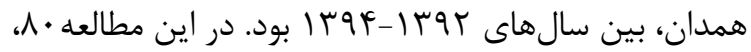

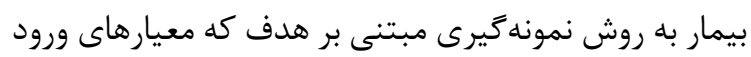
به مطالعه را داشتند، انتخاب و يس از كسب رئي رضايت آكاهانه 
آزمونهاى نايارامترى منويتنى (براى تعيين و مقايسٔ

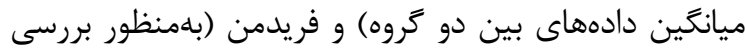

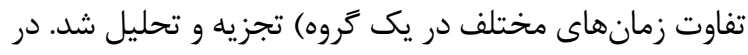
اين مطالعه مبناى معنادارى آمارى هـ/•>-value قرار داده شده است.

\section{بافته ها}

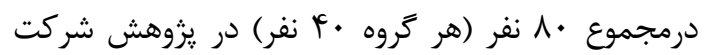

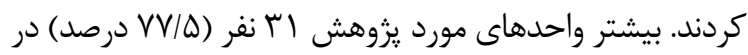
هر دو گروه آزمون و كنترل مرد بودند. ميانكين سنى بيشتر

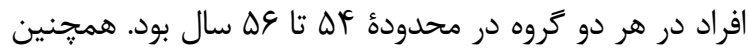
نتايج بيانكر سطح تحصيلات يايين در افراد نمونه است.

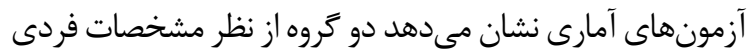
(سن، جنس، تحصيلات، محل زندگى، شاخص توده بدنى،

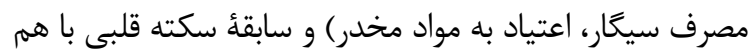

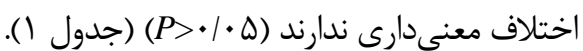

تحصيلات، شاخص توده بدنى، محل زندگى، سابقهُ مصرف

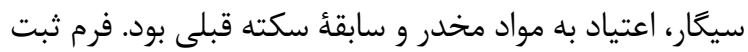
اطلاعات يزشكى شامل كزارش راديولوزى از راديوكرافى قفسئ

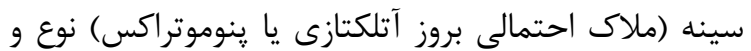

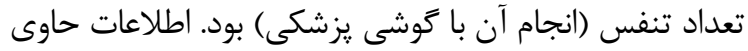

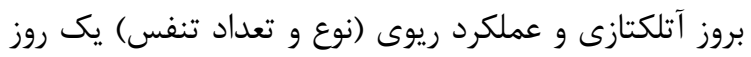

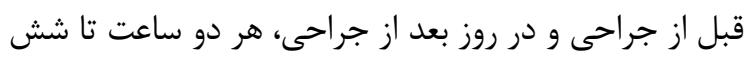

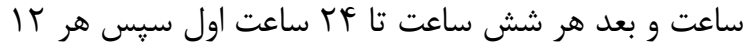

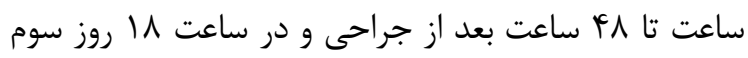

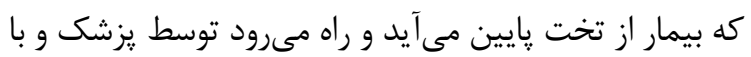
نظارت محقق بررسى و ثبت شد. بايد توجه داشت، راديوگرافى رئ قفسٔ سينه، طبق روتين بخش كرفته شده و هزينهاى از نظر مالى و عوارض جسمى به بيمار تحميل نشده است.

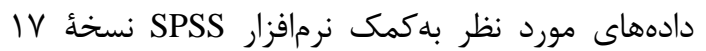
SPSS Inc. Chicago, Illinois, USA) آزمونهاى آمارى توصيفى (ميانگين و انحراف استاندارد) و

جدول ا. مشخصات دموكرافيك در دو كروه آزمون و كنترل

\begin{tabular}{|c|c|c|c|c|}
\hline P-value & تروه كنترل & تروه آزمون & & متغير \\
\hline .1 .9 & $\Delta G / T \Delta(\Delta / F F)$ & $\Delta F / G \wedge(G / F V)$ & & سن/ ميانگين (انحراف ـ \\
\hline \multirow{2}{*}{1} & rI (VV/D) & Wl $(V V / \Delta)$ & مرد & \multirow{2}{*}{ جنس/ تعداد (درصد) } \\
\hline & $q(Y Y / Q)$ & $q(Y Y / Q)$ & 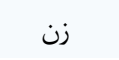 & \\
\hline \multirow{5}{*}{.$/ 49$} & $10(T V / \Delta)$ & $r Y(\Delta \Delta)$ & بىسواد & \multirow{5}{*}{ تحصيلات/ تعداد (درصد) } \\
\hline & $r r(\Delta \Delta)$ & $\mathbb{N}(r \cdot)$ & ابتدايى & \\
\hline & $1(r / \Delta)$ & $r(V / \Delta)$ & ر راهنمايى & \\
\hline & $r(\Delta)$ & $r(\Delta)$ & دبيرستان & \\
\hline & · & $1(r / \Delta)$ & دانشخاهى & \\
\hline \multirow{2}{*}{. } & $r \cdot(\Delta \cdot)$ & $1 \wedge(f \Delta)$ & شهر & \multirow{2}{*}{ محل زندگى / تعداد (درصد) } \\
\hline & $r \cdot(\Delta \cdot)$ & $r r(\Delta \Delta)$ & روستا & \\
\hline \multirow{3}{*}{.1 .99} & $r r(\Delta V / \Delta)$ & $\| \wedge\left(f^{c} \Delta\right)$ & $\leq r \Delta$ & \multirow{3}{*}{ شاخص تودة بدنى/ تعداد (درصد) } \\
\hline & $I V(F / \Delta)$ & $I V(F Y / Q)$ & $r \Delta-r q$ & \\
\hline & $\cdot$ & $\Delta(\mid Y / \Delta)$ & $2 r$. & \\
\hline \multirow{2}{*}{. } & $9(T r / Q)$ & $\mid r(r r / \Delta)$ & 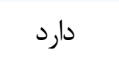 & \multirow{2}{*}{ سابقه مصرف سيگًار / تعداد (درصد) } \\
\hline & rI (VV/D) & $T V(\varphi V / \Delta)$ & 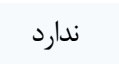 & \\
\hline \multirow{2}{*}{$\cdot / 49$} & $9(1 Q)$ & $9(T r / Q)$ & 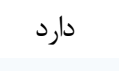 & \multirow{2}{*}{ عتياد به مواد مخدر/ تعداد (درصد) } \\
\hline & $r F(\wedge \Delta)$ & rI $(V V / \Delta)$ & ن ارد & \\
\hline \multirow{2}{*}{. $\mid 94$} & $\mid f(r \Delta)$ & $19(4 \cdot)$ & دارد & \multirow{2}{*}{ سابقة سكته قلبى / تعداد (درصد) } \\
\hline & $r \varepsilon(\& \Delta)$ & TF( (घ)) & 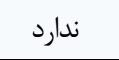 & \\
\hline
\end{tabular}




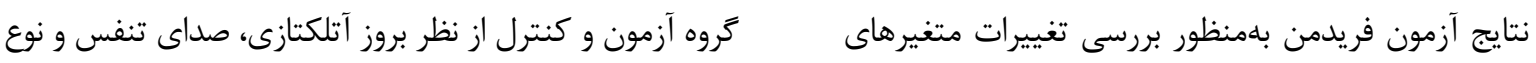

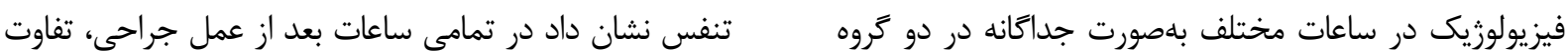

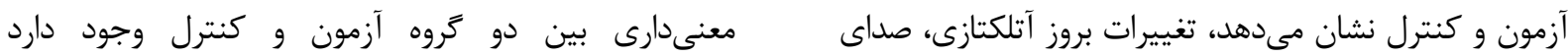

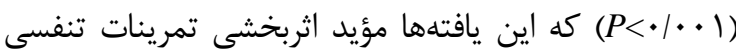

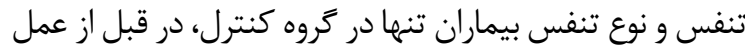

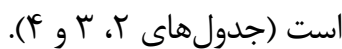

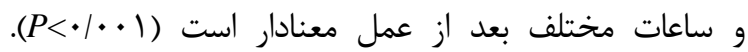

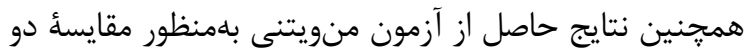

جدول ז. مقايسئ آتلكتازى بين دو كروه آزمون و كنترل در ساعات مختلف (براساس كزارش راديوَّرافى ريه)

\begin{tabular}{|c|c|c|c|c|c|c|}
\hline \multirow[b]{2}{*}{ *P-value } & \multirow{2}{*}{$\begin{array}{c}\text { آماره آزمون } \\
\text { U }\end{array}$} & \multicolumn{2}{|c|}{ تروه كنترل } & \multicolumn{2}{|c|}{ تروه آزمون } & تروه \\
\hline & & تعداد (درصد) & 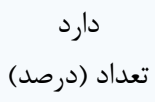 & 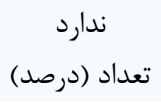 & 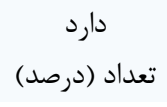 & زآتلكتازى \\
\hline $.1 .9 r$ & $-1 / 99$ & $r \Delta(\Lambda V / \Delta)$ & $\Delta(I Y / \Delta)$ & ५ (qV/D) & $1(T / \Delta)$ & قبل از عمل \\
\hline$<\cdot / \cdot \cdot 1$ & $-\Delta / 9$. & $\|(Y V / \Delta)$ & $r q(V Y / \Delta)$ & $r V(9 Y / D)$ & $r(V / \Delta)$ & r ساعت اول روز اول \\
\hline$<\cdot \mid \cdot \cdot 1$ & $-9 / 49$ & $q(Y Y / Q)$ & M $(V V / \Delta)$ & $r V(9 Y / D)$ & $r(V / \Delta)$ & r ساعت دوم روز اول \\
\hline$<\cdot \mid \cdot \cdot 1$ & $-9 / 49$ & $9(T r / Q)$ & TI (VV/Q) & $r V(9 Y / \Delta)$ & $r(V / \Delta)$ & r ساعت سوم روز اول \\
\hline$<\cdot \mid \cdot \cdot 1$ & $-\Delta / V \cdot$ & $\operatorname{Ir}(r \cdot)$ & $r \wedge(V \cdot)$ & TV $(9 Y / Q)$ & $r(V / \Delta)$ & צ ساعت دوم روز اول \\
\hline$<\cdot \mid \cdot \cdot 1$ & $-G \mid \Delta F$ & $9(Y Y / \Delta)$ & W $(V V / \Delta)$ & ऍ (१८) & $r(\Delta)$ & צ ساعت سوم روز اول \\
\hline$<\cdot / \cdot \cdot 1$ & $-9 / 99$ & $9(10)$ & $\mu F(\wedge \Delta)$ & ऍ (9Q) & $r(\Delta)$ & צ ساعت جهارم روز اول \\
\hline$<\cdot / \cdot \cdot 1$ & $-\Delta / \Lambda \Lambda$ & $9(Y Y / Q)$ & rI $(V V / Q)$ & $r \wedge(9 \Delta)$ & $r(\Delta)$ & ז I ساعت اول روز دوم \\
\hline$<\cdot|\cdot \cdot|$ & $-\varphi / 99$ & $19(F \vee / D)$ & $r I(\Delta r / \Delta)$ & $f \cdot(1 \cdots)$ & • & r I ساعت دوم روز دوم \\
\hline \multirow[t]{4}{*}{$<\cdot 1 \cdot \cdot 1$} & -r/Vr & $\zeta \wedge(\vee \cdot)$ & $\mathbb{R}(r \cdot)$ & $f \cdot(1 \cdots)$ & · & ساعت \| روز سوم \\
\hline & & \multicolumn{2}{|c|}{$11 \varepsilon / \pi V$} & \multicolumn{2}{|c|}{ १/V८ } & 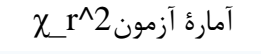 \\
\hline & & \multicolumn{2}{|c|}{9} & \multicolumn{2}{|c|}{9} & درجهأ آزادى \\
\hline & & \multicolumn{2}{|c|}{$<\cdot / \cdot \cdot 1$} & \multicolumn{2}{|c|}{$\cdot / r V$} & $*$ P value \\
\hline
\end{tabular}

* تفاوت بين دو كروه بهكمك آزمون نايارامترى منويتنى' سنجيده شد.

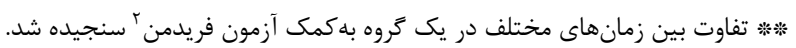

جدول r. مقايسٔ صداى تنفس بين دو كروه آزمون و كنترل در ساعات مختلف روس

\begin{tabular}{|c|c|c|c|c|c|c|}
\hline \multirow{2}{*}{ *P-value } & \multirow{2}{*}{$\begin{array}{c}\text { آمارهُ آزمون } \\
\text { U }\end{array}$} & \multicolumn{2}{|c|}{ تروه كنترل } & \multicolumn{2}{|c|}{ تروه آزمون } & \multirow{2}{*}{ صداى تروه } \\
\hline & & غعداد (درصدي) & تعداد (درصدى) & غعداد (درصد) غيرى & تعداد (درصدي) & \\
\hline .1 .94 & $-1 / 91$ & $1(\Gamma / \Delta)$ & ५ ( (qV/D) & $1(T / \Delta)$ & $r q(q \vee / Q)$ & قبل از عمل \\
\hline$<\cdot / \cdot \cdot 1$ & $-r / 9 \Delta$ & $r(V / \Delta)$ & $r V(9 T / Q)$ & $r(V / \Delta)$ & rV $(9 Y / \Delta)$ & r ساعت اول روز اول \\
\hline$<\cdot / \cdot \cdot 1$ & $-r / r$. & $r(V / \Delta)$ & $r V(9 Y / \Delta)$ & $r(V / \Delta)$ & $r V(q r / Q)$ & r ساعت دوم روز اول \\
\hline$<\cdot / \cdot \cdot 1$ & $-p / \cdot q$ & $f(1 \cdot)$ & एq (q•) & $r(V / \Delta)$ & $r V(q r / Q)$ & r ساعت سوم روز اول \\
\hline$<\cdot / \cdot \cdot 1$ & $-r / v q$ & $r(\Delta)$ & ऍ $(9 \Delta)$ & $1(T / \Delta)$ & $r q(q \vee / Q)$ & צ ساعت دوم روز اول \\
\hline$<\cdot / \cdot \cdot 1$ & $-\Delta / / \Delta$ & $1(T / \Delta)$ & ५ ( (१V/D) & $1(T / \Delta)$ & $r q(q \vee / Q)$ & 4 ساعت سوم روز اول \\
\hline$<\cdot 1 \cdot \cdot 1$ & $-Q / 1 Q$ & $1(T / \Delta)$ & ५ $(9 \vee / Q)$ & $1(\Gamma / \Delta)$ & rq $(9 \vee / 0)$ & צ ساعت جهارم روز اول \\
\hline
\end{tabular}

1. Independent Sample Test U-Mann Whitney

${ }^{2}$. Fridman Test

دورة 


\begin{tabular}{|c|c|c|c|c|c|c|}
\hline \multirow{2}{*}{ *P-value } & \multirow{2}{*}{$\begin{array}{c}\text { آماره آزمون } \\
\text { U }\end{array}$} & \multicolumn{2}{|c|}{ كروه كنترل } & \multicolumn{2}{|c|}{ كروه آزمون } & \multirow{2}{*}{ صداى تروه تنفس } \\
\hline & & غعداد (درصدى) & تعداد (درصدى) & غعداد (درصد) & تعداد (درصد) & \\
\hline$<\cdot / \cdot \cdot 1$ & $-F / \Delta F$ & $r(\Delta)$ & ऍ $(9 \Delta)$ & $r(\Delta)$ & r (१८) & זו ساعت اول روز دوم \\
\hline$<\cdot / \cdot \cdot 1$ & $-r / T \Delta$ & - & $f \cdot(1 \cdots)$ & · & $f \cdot(1 \cdots)$ & ז ساعت دوم روز دوم \\
\hline \multirow[t]{4}{*}{$<\cdot 1 \cdot \cdot 1$} & 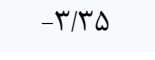 & - & $r \cdot(1 \cdots)$ & $\cdot$ & $f \cdot(1 \cdots)$ & ساعت \1 روز سوم \\
\hline & & \multicolumn{2}{|c|}{$99 / 94$} & \multicolumn{2}{|c|}{$\mid r / 9}$. & آمارة آزمون2r \\
\hline & & \multicolumn{2}{|c|}{9} & \multicolumn{2}{|c|}{9} & مرجهُ آزادى \\
\hline & & \multicolumn{2}{|c|}{$<\cdot 1 \cdot \cdot 1$} & \multicolumn{2}{|c|}{$\cdot / 1 \Lambda$} & $*$ P value \\
\hline
\end{tabular}

* تفاوت بين دو كروه با استفاده از آزمون نايارامترى منويتنى سنجيده شد.

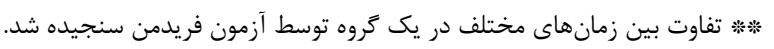

\begin{tabular}{|c|c|c|c|c|c|c|c|c|}
\hline \multirow[b]{2}{*}{ *P-value } & \multirow{2}{*}{$\begin{array}{c}\text { آمارهُ } \\
\text { U }\end{array}$} & \multicolumn{3}{|c|}{ كروه كنترل } & \multicolumn{3}{|c|}{ تروه آزمون } & \multirow{2}{*}{ 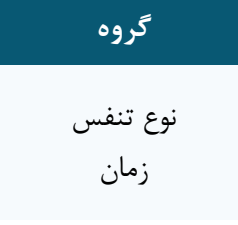 } \\
\hline & & با زحمت & ديافراگمى & سطعى سعداد & با زحمت & ديافراگمى & سعرادى & \\
\hline$\cdot / \cdot \cdot 1$ & $-r / \Delta 9$ & $\cdot$ & $r r(\Delta V / \Delta)$ & $I V(F T / Q)$ & $\cdot$ & $r V(9 T / Q)$ & $r(V / Q)$ & قبل از عمل \\
\hline • & $-r / \cdot r$ & $f(1 \cdot)$ & $\mid r(\Psi r / \Delta)$ & $r r(\Delta V / \Delta)$ & $r(V / \Delta)$ & $r \cdot(v \Delta)$ & $V(I V / Q)$ & r ساعت اول روز اول \\
\hline$\cdot 1 \cdot \cdot 1$ & -r/fr & $r(\Delta)$ & $1 \Delta(r V / \Delta)$ & $r r(\Delta V / \Delta)$ & $r(V / \Delta)$ & $r \cdot(V \Delta)$ & $V(I V / Q)$ & r ساعت دوم روز اول \\
\hline$<\cdot / \cdot \cdot 1$ & $-r / \Delta V$ & $1(T / Q)$ & $19(f \cdot)$ & $r(\Delta V / \Delta)$ & $r(\Delta)$ & r $(V V / Q)$ & $V(I V / \Delta)$ & r ساعت سوم روز اول \\
\hline$<\cdot \mid \cdot \cdot 1$ & $-r|\Lambda|$ & $1(Y / \Delta)$ & $19(1 \cdot)$ & $r r(\Delta V / \Delta)$ & $r(\Delta)$ & rT $(\Lambda \cdot)$ & $q(10)$ & צ ساعت دوم روز اول \\
\hline$<\cdot / \cdot \cdot 1$ & $-F / \Delta \varphi$ & $I V(F Y / D)$ & IV $(F T / Q)$ & $r(\Delta V / \Delta)$ & $r(\Delta)$ & $\operatorname{rF}(\wedge \Delta)$ & $f(1 \cdot)$ & צ ساعت سوم روز اول \\
\hline$<\cdot / \cdot \cdot 1$ & $-F / \Lambda \Lambda$ & $\cdot$ & $1 \wedge\left(\varphi^{F} \Delta\right)$ & $T r(\Delta \Delta)$ & $1(T / \Delta)$ & $r V(9 T / Q)$ & $r(\Delta)$ & צ ساعت جهارم روز \\
\hline$<\cdot \mid \cdot \cdot 1$ & $-r / \Delta T$ & $\cdot$ & $r \cdot(\Delta \cdot)$ & $r \cdot(\Delta \cdot)$ & $1(Y / \Delta)$ & $r V(9 T / Q)$ & $r(\Delta)$ & rاعت اول سوم روز \\
\hline$<\cdot / \cdot \cdot 1$ & $-r / V q$ & $\cdot$ & $r \varepsilon(\varepsilon \Delta)$ & If $(r \Delta)$ & $1(T / \Delta)$ & $r \wedge(9 \Delta)$ & $1(T / Q)$ & ז داعت دوم روز \\
\hline \multirow[t]{4}{*}{$<\cdot 1 \cdot \cdot 1$} & 每 & · & rq $(V T / \Delta)$ & $\|(T V / Q)$ & $1(T / \Delta)$ & $r \wedge(१ \Delta)$ & $1(T / Q)$ & ساعت \\
ا روز سوم \\
\hline & & & $\forall \& / 4 \Delta$ & & & $|r / 4|$ & & آمارؤ آزمون2 \\
\hline & & & 9 & & & 9 & & درجهُ آزادى \\
\hline & & & $<\cdot / \cdot \cdot 1$ & & & $\cdot / 1 F$ & & $* * P$ value \\
\hline
\end{tabular}

* تفاوت بين دو كروه توسط آزمون نايارامترى منويتنى سنجيده شد.

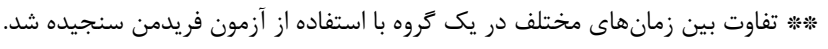

بيمارستان انجام مىشود، در كاهش آتلكتازى بعد از جراحى

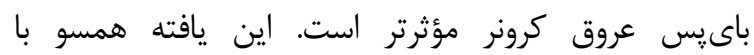
مطالعة Shaban و همكاران است كه بيان مىكنيند تمرينات

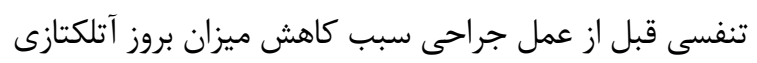

نتايج مطالعهُ حاضر نشان مىدهد تمرينات تنفسى شامل

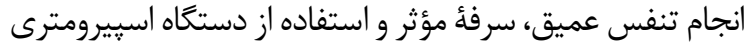

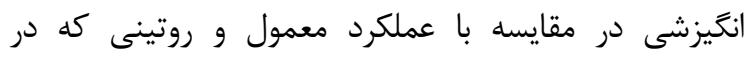




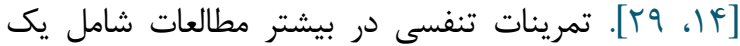

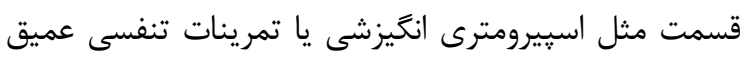
بوده و از يك بروتكل تركيبى شامل تنفس عميق، سرفه مؤثر و

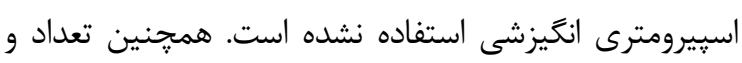
دفعات انجام تمرينات تنفسى نقش مهمى در كاهش عوارض

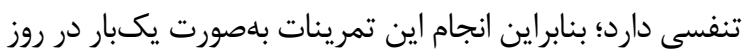
ممكن است سبب تغييرات مثبت بالينى نشود. تغييرات ايجادشده در روند اكسيزنرسانى بعد از تمرينات تنفسى موقتى

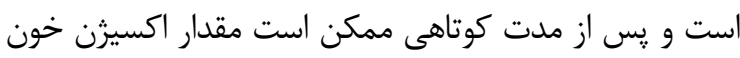

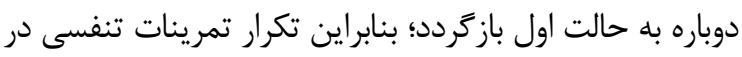

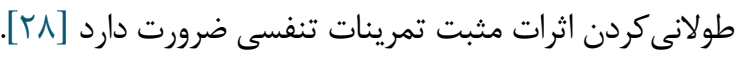

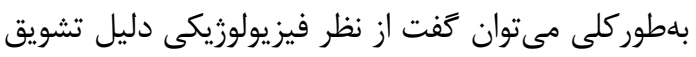
به انجام تمرينات تنفس عميق اين است كه اين تمرينات سبب

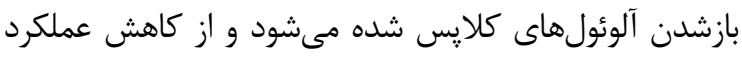

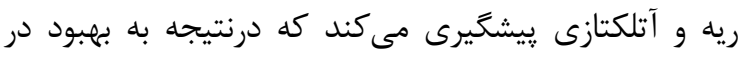
نسبت تهويه و يرفيوزن مىانجامد. اين تمرينات درواقع ميزان

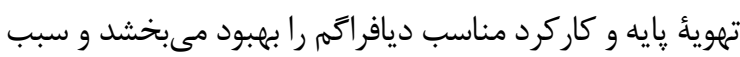

بهبود حجم جارى و تسهيل خروج ترشحات مىشود [•r"].

\section{كاربرد يافتههاى يخوهش در بالين}

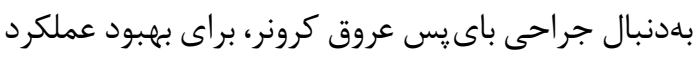

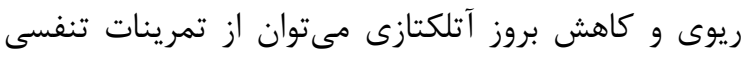

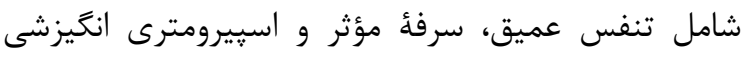

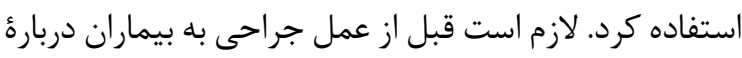

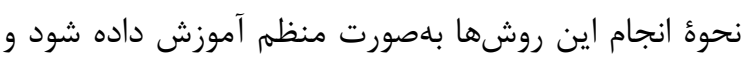
انجام آنها بعد از عمل نيز بهصورت منسجم و و سازمانيان منافته

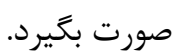

\section{نتيجه گيرى}

نتايج حاصل از مطالعأ حاضر نشان مىدهد تمرينات تنفسى شامل انجام تنفس عميق، سرفئ مؤثر و استفاده از دستخاه اسيِيرومترى انكَيزشى در مقايسه با عملكرد معمول

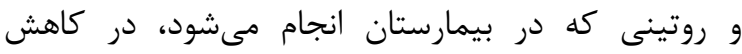

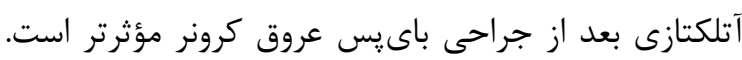
اين مطالعه دربارهٔ بيمارانى كه مشكلات ريوى ندائ نداشتند انجام

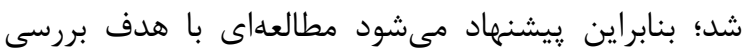

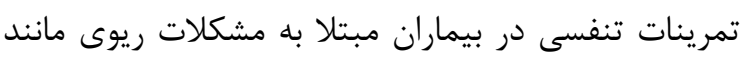
انسداد مزمن راههاى هوايى و آسم نيز انجام شود.
و بهبود وضعيت تهويه مىشود [rT]. Shakouri وكمكاران مطالعهاى را با عنوان تأثير آموزش به كاركيرى تمرينات تنفسى إنى إنى

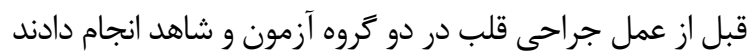

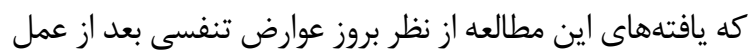

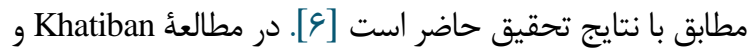

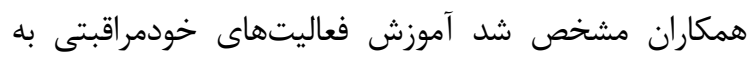

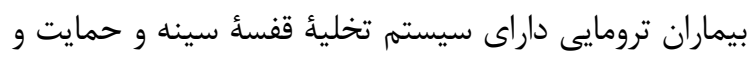
تشويق به انجام اين فعاليتها در ويشخَيرى از آتلكتازى آنها

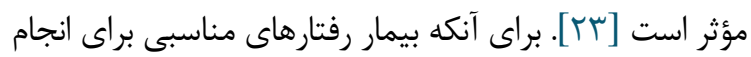

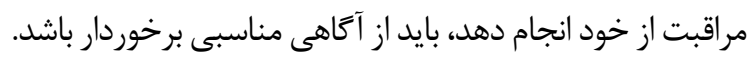
در مطالعة Oshvandi و همكاران نيز توان خودمراقبتى در بيماران گَروه مداخله بهطور معنى دارى از گروه كنترل بهتر بود

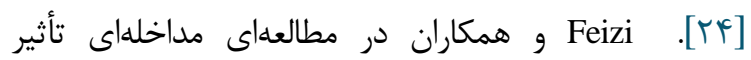
اسييرومترى انكَيزشى و تمرينات تنفسى عميق را بر كازخون

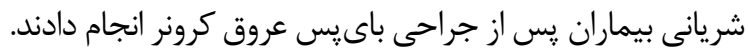

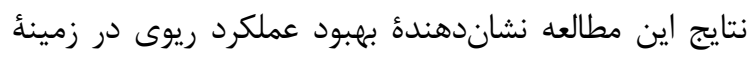

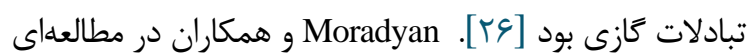
مداخلهاى، تأثير تمرينات تنفسى برنامهريزىشده رأ برات

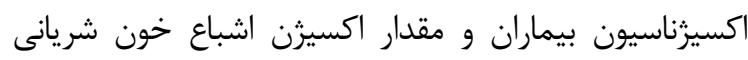
بررسى كردند. بهمنظور سنجش عملكرد ريوى اطلاعات مربوط به كازخون شريانى و اشباع اكسيرن جمعآورى شدرى شد و نتايج مطالعه نشاندهندة روند بهتر بهبود اكسيزناسيون بيماران بود،

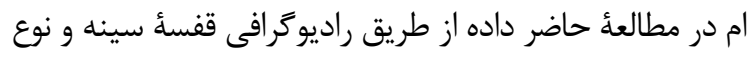
و تعداد تنفس ميزان بروز آتلكتازى مورد بررسى قرار كرفيته

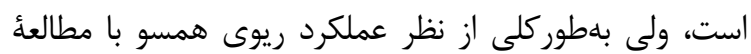
حاضر است [TV] همجنين نتايج اين مطالعه نشان مىدهد در كروه كن كنترل تغييرات محسوسى در عملكرد رئُ بيماران بهصورت آتلكتازى،

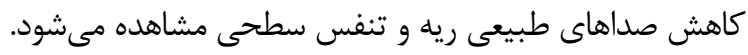

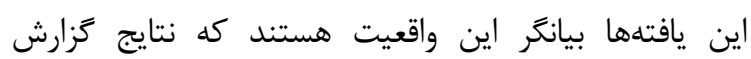
راديوكرافى، صدا و نوع تنفس بيماران تحت تأثير تمرينات تنفسى مرتب و اصولى تغيير مى كند. اين يافتهها با مطالعات

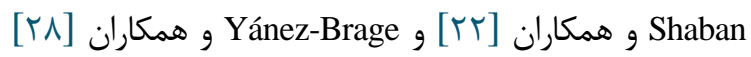

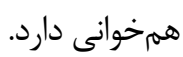
كزارش مطالعات مرتبط با اثرات تمرينات تنفسى متفاوت

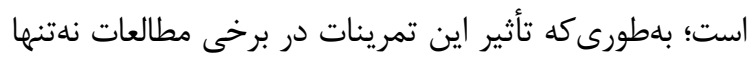

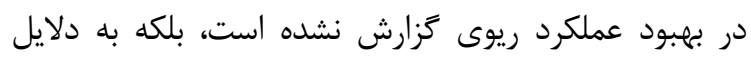
متعدد، اثرات منفى براى انجام تمرينات تنفسى ذكر كردماند برداند 


$$
\begin{aligned}
& \text { فرزانه رشيدى فكارى و همكاران qr q4 } \\
& \text { آنها در انجام يزوهش و از بيماران محترم به دليل شركت } \\
& \text { در اين مطالعه اعلام مى كنند. }
\end{aligned}
$$

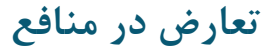

$$
\begin{aligned}
& \text { بين نويسندكان هيجَّّنه تعارضى در منافع وجود ندارد }
\end{aligned}
$$

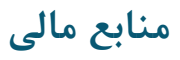

$$
\begin{aligned}
& \text { منابع مالى اين مطالعه توسط نويسندكان تامين شده است. } \\
& \text { سياسگزارى } \\
& \text { اين مقاله بخشى از ياياننامه كارشناسى ارشد يرستارى }
\end{aligned}
$$

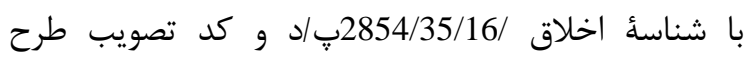

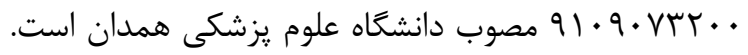

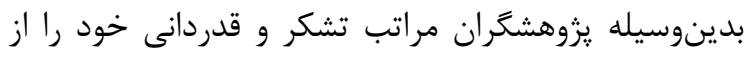

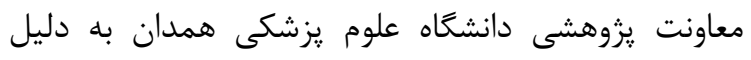

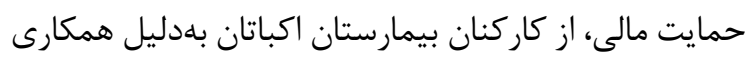

\section{References}

1. Keenan TD, Abu-Omar Y, Taggart DP. Bypassing the pump: changing practices in coronary artery surgery. CHEST Journal. 2005;128(1):363-9. [DOI:10.1378/chest.128.1.363] [PMID]

2. Santos NPd, Mitsunaga RM, Borges DL, Costa MdAG, Baldez TEP, Lima IM, et al. Factors associated to hypoxemia in patients undergoing coronary artery bypass grafting. Brazilian Journal of Cardiovascular Surgery. 2013;28(3):364-70. [DOI:10.5935/1678-9741.20130056] [PMID]

3. Pasquina P, Walder B. Prophylactic respiratory physiotherapy after cardiac surgery: systematic review. Bmj. 2003;327(7428):1379 [DOI:10.1136/bmj.327.7428.1379] [PMID] [PMCID]

4. Chiarenza F, Tsoutsouras T, Cassisi C, Santonocito C, Gerry S, Astuto M, et al. The Effects of On-Pump and OffPump Coronary Artery Bypass Surgery on Respiratory Function in the Early Postoperative Period. Journal of intensive care medicine. 2017:0885066617696852. [DOI:10.1177/0885066617696852] [PMID]

5. Noppen M, De Keukeleire T. Pneumothorax. Respiration. 2008;76(2):121-7. [DOI:10.1159/000135932] [PMID]

6. Shakouri SK, Salekzamani Y, Taghizadieh A, SabbaghJadid H, Soleymani J, Sahebi L, et al. Effect of respiratory rehabilitation before open cardiac surgery on respiratory function: a randomized clinical trial. Journal of cardiovascular and thoracic research. 2015;7(1):13. [DOI:10.15171/jcvtr.2014.03] [PMID] [PMCID]

7. Asgari P, Bahramnezhad F, Mehrdad N, Noughabi AAA, Hekmatpou D, Mahmoudi M. Depression, functionality and adaptability of elderly patients after open heart surgery offor on-pump. Jundishapur Journal of Chronic Disease Care. 2015;4(4). [DOI:10.17795/jijcdc-30508]

8. Moradi B, Teymouri H, Porya A, Ebrahimzadeh F. The effect of two different levels of positive end expiratory pressure (PEEP) in the incidence of atelectasis after coronary artery bypass graft surgery. Yafte. 2017;19(2).

9. Zaky A, Lang JD. The use of intraoperative positive end expiratory pressure. J Anesth Clin Res. 2011;3:002.

10. Lagow EE, Leeper BB, Jennings LW, Ramsay MA, editors. Incidence and severity of respiratory insufficiency detected by transcutaneous carbon dioxide monitoring after cardiac

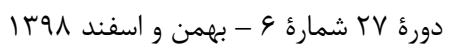

surgery and intensive care unit discharge. Baylor University Medical Center Proceedings; 2013: Taylor \& Francis. [DOI:10.1080/08998280.2013.11929009] [PMID] [PMCID]

11. Renault JA, Costa-Val R, Rosseti MB, Houri Neto M. Comparison between deep breathing exercises and incentive spirometry after CABG surgery. Brazilian Journal of Cardiovascular $\quad$ Surgery. 2009;24(2):165-72. [DOI:10.1590/S0102-76382009000200012] [PMID]

12. Gilani SRA, Hussain G, Ahmad N, Baig MAR, Zaman H. COMPARISON OF POST-OPERATIVE ATELECTASIS IN PATIENTS UNDERGOING CORONARY ARTERY BYPASS GRAFTING WITH AND WITHOUT PREOPERATIVE INCEN-TIVE SPIROMETRY. JPMI. 2016;30(2):169.

13. Soman A, Mundyat G, Kumar D, Santhakumar H. Does body mass index influence pulmonary function test values and functional exercise capacity after chest physiotherapy following coronary artery bypass graft. Indian Journal of Thoracic and Cardiovascular Surgery. 2017:1-9. [DOI:10.1007/s12055-017-0528-8]

14. Westerdahl E, Lindmark B, Eriksson T, Friberg O, Hedenstierna G, Tenling A. Deep-breathing exercises reduce atelectasis and improve pulmonary function after coronary artery bypass surgery. CHEST Journal. 2005;128(5):3482-8. [DOI:10.1378/chest.128.5.3482] [PMID]

15. Lalegani H, Esmaili Vardanjani S, Safdari A. The effects of breathing techniques on pain intensity of burn dressing. Journal of Clinical Nursing and Midwifery. 2014;2.

16. Herdy AH, Marcchi PL, Vila A, Tavares C, Collaço J, Niebauer $\mathbf{J}$, et al. Pre-and postoperative cardiopulmonary rehabilitation in hospitalized patients undergoing coronary artery bypass surgery: a randomized controlled trial. American journal of physical medicine \& rehabilitation. 2008;87(9):714-9. [DOI:10.1097/PHM.0b013e3181839152] [PMID]

17. Fiore JF, Chiavegato LD, Denehy L, Paisani DM, Faresin SM. Do directed cough maneuvers improve cough effectiveness in the early period after open heart surgery? Effect of thoracic support and maximal inspiration on cough peak expiratory flow, cough expiratory volume, and thoracic pain. Respiratory care. 2008;53(8):1027-34.

18. Hulzebos EH, Helders PJ, Favié NJ, De Bie RA, de la Riviere AB, Van Meeteren NL. Preoperative intensive

$$
\text { مجله مراقبت برستارى و مامايى ابنسينا }
$$


inspiratory muscle training to prevent postoperative pulmonary complications in high-risk patients undergoing CABG surgery: a randomized clinical trial. Jama. 2006;296(15):1851-7. [DOI:10.1001/jama.296.15.1851] [PMID]

19. Stannard D. Incentive Spirometry for Preventing Pulmonary Complications After Coronary Artery Bypass Graft. Journal of PeriAnesthesia Nursing. 2013;28(4):236-8. [DOI:10.1016/j.jopan.2013.05.003] [PMID]

20. Feizi h, mohammadi h, yazdannik a, mir mohammad sadeghi $m$, zamani p. Effect of incentive spirometry and deep breathing exercises on arterial blood gas parameters after coronary artery bypass graft surgery. Cardiovascular Nursing Journal. 2016;5(3):52-8.

21. Moradyan T, Farahani M, Mohammadi N, Jamshidi R. The effect of planned breathing exercises on oxygenation in patients after coronary artery bypass surgery. Cardiovascular Nursing Journal. 2012;1(1):8-14.

22. Shaban M, Salsali M, Kamali P, Poormirzakalhori R. Assessment the effects of respiratory exercise education in acute respiratory complication and the length of patient hospitalization, for undergoing coronary artery by-pass surgery in Kermanshah Emam Ali hospital. Journal of hayat. 2002;8(2):12-20.

23. Khatiban M, Shirani F, Oshvandi K, Soltanian A, Ebrahimiyan R. Effect of Supportive-Educative Nursing System on Self-Care Skills in Trauma Patients with Chest Drainage System. Journal of hayat. 2014;20(1):48-58.

24. Oshvandi K, Keshmiri K, Salavati M, Emkanjoo Z, Musavi S. Effectiveness of education based on Orem's Self-Care model in Self-Care activity of patients with implantable cardioverter defibrillators. Hayat. 2014;19(3):47-55.

25. Bikmoradi A, Zafari A, Oshvandi K, Mazdeh M, Roshanaei G. Effect of progressive muscle relaxation on severity of pain in patients with multiple sclerosis: a randomized controlled trial. Journal of hayat. 2014;20(1):26-37.

26. FEIZI $\mathrm{H}$, MOHAMMADI $\mathrm{H}$, YAZDANNIK A, MIRMOHAMMAD SM, ZAMANI P. EFFECT OF INCENTIVE SPIROMETRY AND DEEP BREATHING EXERCISES ON ARTERIAL BLOOD GAS PARAMETERS AFTER CORONARY ARTERY BYPASS GRAFT SURGERY. 2016

27. Moradyan T, Farahani M, Mohammadi N, Jamshidi R. The effect of planned breathing exercises on oxygenation in patients after coronary artery bypass surgery. Iranian Journal of Cardiovascular Nursing. 2012;1(1):8-14.

28. Yánez-Brage I, Pita-Fernández S, Juffé-Stein A, MartínezGonzález U, Pértega-Díaz S, Mauleón-García Á. Respiratory physiotherapy and incidence of pulmonary complications in off-pump coronary artery bypass graft surgery: an observational follow-up study. BMC pulmonary medicine. 2009;9(1):36. [DOI:10.1186/1471-2466-9-36] [PMID] [PMCID]

29. Tyler M, Hudson L, Grose B, Huseby J. Prediction of oxygenation during chest physiotherapy in critically ill patients. Am Rev Respir Dis. 1980;121(4 Part 2):218.

30. Hough A. Physiotherapy in respiratory care: an evidencebased approach to respiratory and cardiac management: Nelson Thornes; 2001. 